Cahiers
de la Recherche
sur les Droits

Cahiers de la recherche sur les droits fondamentaux

16 | 2018

Les partis politiques

\title{
Les partis politiques devant la Cour interaméricaine des droits de l'homme
}

Political Parties before the Inter-American Court of Human Rights

\section{Marie Rota}

\section{(2) OpenEdition}

Édition électronique

URL : https://journals.openedition.org/crdf/325

DOI : $10.4000 /$ crdf.325

ISSN : 2264-1246

Éditeur

Presses universitaires de Caen

Édition imprimée

Date de publication : 16 novembre 2018

Pagination : 111-120

ISBN : 978-2-84133-901-3

ISSN : $1634-8842$

Référence électronique

Marie Rota, « Les partis politiques devant la Cour interaméricaine des droits de l'homme », Cahiers de la recherche sur les droits fondamentaux [En ligne], 16 | 2018, mis en ligne le 16 novembre 2019,

consulté le 14 novembre 2022. URL : http://journals.openedition.org/crdf/325 ; DOI : https://doi.org/ $10.4000 /$ crdf.325 


\title{
Les partis politiques devant la Cour interaméricaine des droits de l'homme
}

\author{
Marie ROTA \\ Maître de conférences en droit public à l'université de Lorraine \\ Institut de recherches sur l'évolution de la nation et de l'État (IRENEE)
}

\begin{abstract}
I. La reconnaissance implicite de la licéité des candidatures indépendantes
II. La reconnaissance d'autres formes d'organisation politique aussi légitimes que les partis

III. L'importation annoncée du concept de démocratie militante
\end{abstract}

Les partis politiques du continent américain sont a priori dans une situation privilégiée vis-à-vis de celle dans laquelle se trouvent ceux du continent européen au regard du système de protection régionale des droits et libertés mis en place. Notons tout d'abord que l'article 16 de la Convention américaine relative aux droits de l'homme ${ }^{1}$ mentionne explicitement que toute personne «a le droit de s'associer librement à d'autres à des fins [...] politiques». Là où la Convention de sauvegarde des droits de l'homme et des libertés fondamentales ${ }^{2}$ ne fait que mentionner la liberté de s'associer à des fins syndicales, nécessitant une interprétation dynamique de l'article 11 par la Cour européenne des droits de l'homme ${ }^{3}$ pour y inclure la protection des partis politiques ${ }^{4}$, la Cour interaméricaine des droits de l'homme $^{5}$ a pu sans peine y procéder ${ }^{6}$. Notons en outre que les rédacteurs de la Convention américaine, n'hésitant pas, contrairement à ceux de la Convention européenne, à consacrer le volet institutionnel de la notion de démocratie $^{7}$, ont inséré dans son texte même une disposition consacrant les droits politiques. Son article 23 est de surcroît beaucoup plus détaillé que son équivalent européen, d'où une assise textuelle bien plus intéressante mise à la disposition des partis politiques.

Leur accès à la Cour interaméricaine est en outre facilité par l'existence d'un véritable «droit de dénon-

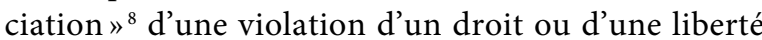
reconnu(e) par la Convention. D'après son article 44 : «Toute personne ou tout groupe de personnes, toute entité non gouvernementale et légalement reconnue dans un ou plusieurs États membres de l'Organisation» a la possibilité de «soumettre à la Commission des pétitions contenant des dénonciations ou plaintes relatives à une

1. Ci-après «Convention américaine» ou «Pacte de San José».

2. Ci-après "Convention européenne».

3. Ci-après «Cour européenne», «Cour de Strasbourg» ou encore «Cour EDH».

4. Sur ce point, voir Y. Lécuyer, L'européanisation des standards démocratiques, Rennes, Presses universitaires de Rennes, 2011, p. 150-153.

5. Ci-après «Cour interaméricaine», "Cour de San José» ou encore «Cour IDH».

6. Cour IDH, Manuel Cepeda Vargas c. Colombie, exceptions préliminaires, fond, réparations et coûts, 26 mai 2010 , série C, nº $213, \$ 172$. Voir aussi Cour IDH, Baena Ricardo et autres c. Panama, fond, réparations et coûts, 2 février 2001, série C, $\mathrm{n}^{\circ} 72, \$ 156$ ou Cour IDH, Escher et autres c. Brésil, exceptions préliminaires, fond, réparations et coûts, 6 juillet 2009, série C, nº 200, $\$ 170$ ou encore Cour IDH, Kawas Fernández c. Honduras, fond, réparations et coûts, 3 avril 2009, série $C, n^{\circ} 196, \$ 143$.

7. Voir M. Rota, L'interprétation des Conventions américaine et européenne des droits de l'homme. Analyse comparée de la jurisprudence des Cours européenne et interaméricaine des droits de l'homme, Issy-les-Moulineaux, LGDJ, 2018, p. 408-409.

8. H. Tigroudja, I. K. Panoussis, La Cour interaméricaine des droits de l'homme. Analyse de la jurisprudence consultative et contentieuse, Bruxelles, Bruylant, 2003, p. 105 
violation de la présente Convention par un État partie». La faculté de déposer une pétition devant la Commission n'est donc pas conditionnée par la qualité de victime de son auteur et ce dernier n'a en outre pas toujours besoin de l'autorisation des victimes pour pouvoir agir ${ }^{9}$. De nombreuses associations se saisissent d'ailleurs de cette voie de recours pour faire valoir les droits des victimes présumées ${ }^{10}$.

Il ne semble en revanche pas que ce soit le cas s'agissant des partis politiques. Dans les quelques affaires les concernant, ce sont en effet principalement leurs membres qui saisissent la Commission lorsqu'ils estiment que leurs droits et libertés sont violés. Cela peut peut-être s'expliquer par le fait que les personnes morales ne peuvent pas être reconnues comme victimes d'une violation de la Convention. La Cour, invoquant des motifs de sécurité juridique ${ }^{11}$, soumet en effet la recevabilité de la pétition à l'existence d'une victime dûment identifiée et individualisée dans la demande ${ }^{12}$. Or, une personne morale ne peut, selon elle, être qualifiée comme telle, puisqu'elle ne saurait être reconnue comme titulaire de droits et libertés ${ }^{13}$.

Notons cependant qu'elle reconnaît depuis fort longtemps qu'un "groupe de personnes" puisse l'être. C'est le cas des populations autochtones mais aussi des migrants ${ }^{14}$, des femmes ${ }^{15}$ ou encore des homosexuels ${ }^{16}$. On peut alors se demander si un parti politique pourrait être reconnu comme groupe titulaire de droits et libertés, au-delà de sa qualification de personne morale. Pour répondre à cette question, encore faut-il rappeler que la détermination de l'existence d'un groupe découle de l'application du principe de non-discrimination. Il faut aussi avoir à l'esprit que la dignité de la personne humaine est étroitement reliée au principe d'égalité, qui en est, selon la Cour, son « expression première ${ }^{17}$. Toute personne doit donc pouvoir bénéficier des droits et libertés de manière égale et ce n'est que si un groupe de personnes fait l'objet de discrimination dans leur jouissance ${ }^{18}$ qu'il pourra se voir octroyer une protection particulière.

Mais, à partir de là, pourrait-on alors envisager qu'un parti politique représentant des minorités nationales puisse l'être? Il faut en douter. Seule une affaire concerne en effet indirectement ce cas de figure: l'affaire Yatama c. Nicaragua relative à un parti politique représentant une communauté autochtone. Or, dans cette affaire, c'est la communauté en tant que telle qui a été reconnue comme groupe méritant respect. Les violations constatées ne le sont pas en raison de l'appartenance des victimes au parti YATAMA mais en raison de leur appartenance à cette minorité $^{19}$. La formation politique en cause ne saurait donc être reconnue comme victime d'une violation de droits et libertés conventionnellement protégés. Et si la Cour accepte de lui accorder une réparation au titre du préjudice moral, ce n'est que celui subi par ses membres qui est pris en considération, nécessitant, de fait, une redistribution de la somme accordée aux parties lésées ${ }^{20}$.

On pourrait en revanche imaginer une situation dans laquelle les membres d'un parti politique seraient discriminés dans la jouissance de leurs droits et libertés en raison de

9. Dans l'affaire Yatama c. Nicaragua, par exemple, la Cour reconnaît que les représentants des victimes agissent également au nom de celles qui n'ont pas donné leur pouvoir; Cour IDH, Yatama c. Nicaragua, exceptions préliminaires, fond, réparations et coûts, 23 juin 2005, série C, $\mathrm{n}^{\circ} 127, \$ 92$.

10. Voir M. Rota, L'interprétation des Conventions..., p. 167.

11. Cour IDH, Radilla Pacheco c. Mexique, exceptions préliminaires, fond, réparations et coûts, 23 novembre 2009 , série C, $n^{\circ} 209$, $\$ 110$.

12. Cour IDH, Instituto de Reeducación del Menor c. Paraguay, exceptions préliminaires, fond, réparations et coûts, 2 septembre 2004 , série C, $\mathrm{n}^{\circ}$ 112, $\$$ 109. L'article 35 du règlement de la Cour prévoit en outre que: «L'affaire est déposée devant la Cour par la remise du rapport visé à l'article 50 de la Convention, qui contient tous les faits présumés en cause, y compris l'identification des victimes présumées» (nous soulignons). Il faut cependant préciser que la Commission ne requiert pas du pétitionnaire une telle identification: l'article $28-5$ du règlement de la Commission prévoit que la pétition doit comporter «si possible, le nom de la victime». Sur cette exigence, voir D. Rodríguez Pinzón, "The "Victim" Requirement, the Fourth Instance Formula and the Notion of "Person" in the Individual Complaint Procedure of the Inter-American Human Rights System", International Law Student Association Journal of International and Comparative Law, no 7, 2001, p. 373-388.

13. La Cour a d'ailleurs confirmé cette approche dans son opinion consultative, Cour IDH, Titularidad de derechos de las personas jurídicas en el sistema interamericano de derechos humanos (Interpretación y alcance del artículo 1.2, en relación con los artículos 1.1, 8, 11.2, 13, 16, 21, 24, 25, 29, 30, 44, 46, y 62.3 de la Convención Americana sobre Derechos Humanos, así como del artículo 8.1 A y B del Protocolo de San Salvador), opinion consultative $\mathrm{n}^{\circ} \mathrm{OC}-22 / 16,26$ février 2016, série $A, \mathrm{n}^{\circ}$ 22. Mobilisant tout l'éventail des techniques d'interprétation proposées par le droit international, elle affirme que les personnes morales ne peuvent, par principe, être reconnues comme titulaires de droit, au sens de la Convention.

14. Voir, par exemple, Cour IDH, Vélez Loor c. Panama, exceptions préliminaires, fond, réparations et coûts, 23 novembre 2010 , série C, ${ }^{\circ} 218$, $\$ 99$.

15. Voir, par exemple, Cour IDH, González et autres (“Campo Algodonero") c. Mexique, exceptions préliminaires, fond, réparations et coûts, 16 novembre 2009, série C, n 205. Voir aussi le commentaire de L. Burgorgue-Larsen, A. Úbeda de Torres, "Women's Rights », in The Inter-American Court of Human Rights: Case Law and Commentary, L. Burgorgue-Larsen, A. Úbeda de Torres (dir.), Oxford, Oxford University Press, 2011, p. 411-452.

16. Cour IDH, Atala Riffo y niñas $c$. Chili, fond, réparations et coûts, 24 février 2012, série $\mathrm{C}, \mathrm{n}^{\circ} 239, \S 79$. Il est à cet égard tout à fait remarquable d'observer son changement de terminologie dans cette affaire puisqu'elle y affirme explicitement que toute situation qui conduit à traiter de manière discriminatoire « un groupe» - et non plus une personne en raison de l'appartenance à ce groupe - enfreint l'article 1.1 de la Convention ( $i b i d$.)

17. M. Rota, L'interprétation des Conventions..., p. 363. Voir, entre autres, Cour IDH, Condición jurídica y derechos de los migrantes indocumentados, opinion consultative $\mathrm{n}^{\circ} \mathrm{OC}-18 / 03,17$ septembre 2003 , série $\mathrm{A}, \mathrm{n}^{\circ} 18, \S 100$.

18. Dans l'affaire Atala Riffo y niñas c. Chili, la Cour affirme à ce titre que «la notion d'égalité est inséparable de la dignité essentielle de la personne, avec laquelle est incompatible toute situation qui conduit à traiter un groupe déterminé de manière privilégiée du fait de la reconnaissance de sa supériorité; ou qui conduit, à l'inverse, à le traiter avec hostilité ou de façon à le discriminer dans la jouissance des droits qui sont quant à eux reconnus à ceux qui ne se trouvent pas dans une telle situation, puisque considérés comme inférieurs» ( $\$ 79)$. Sauf indication contraire, les traductions des décisions de la Cour interaméricaine sont celles de l'auteure.

19. Il semble d'ailleurs que la Cour interaméricaine entende davantage le terme de "groupe» comme renvoyant au concept de «minorité» tel que développé par Yaël Attal-Galy par exemple (Y. Attal-Galy, Droits de l'homme et catégories d'individus, Paris, LGDJ, 2003, p. 19).

20. La Cour accepte en effet de verser une indemnité à YATAMA mais pour qu'elle la répartisse ensuite entre ses membres lésés (Cour IDH, Yatama c. Nicaragua, $\$ 248)$ 
leur appartenance réelle suspectée audit parti. Dans cette hypothèse, faisant écho à de nombreuses situations déjà examinées par la Cour dans des contextes dictatoriaux ou de guérillas ${ }^{21}$, se pourrait-il que le parti politique en cause puisse être reconnu comme titulaire de droits et libertés? Si leur violation ne pourrait fonder une pétition devant la Commission, puisqu'un parti ne peut, en tant que personne morale, être reconnu comme victime, le parti pourrait avoir tout intérêt à exercer son droit de dénonciation à l'égard de la violation des droits et libertés de ses membres ou au moins à s'associer à une procédure en cours en vue de faire valoir la violation de ses droits collectifs et obtenir éventuellement réparation.

À cet égard, la Cour a déjà par exemple reconnu la nature «duale» de certains droits et libertés, tels que la liberté d'expression ou encore la liberté d'association ${ }^{22}$. Dans une récente affaire relative aux organisations syndicales, elle souligne que «les droits qui dérivent de la représentation des intérêts d'un groupe ont une nature duale» dans la mesure où ils se manifestent à la fois dans «le droit de l'individu» qui a été élu à une charge électorale ainsi que «dans le droit de la collectivité d'être représentée ${ }^{23}$. En effet, selon elle «la violation du droit de ce premier (le représentant) implique l'affaiblissement du droit de l'autre (le représenté) ${ }^{24}$. Dans l'affaire Chitay Nech et autres c. Guatemala, elle avait d'ailleurs déjà reconnu que la disparition forcée d'un membre d'une communauté autochtone qui avait été élu maire d'une commune violait non seulement ses droits politiques mais aussi ceux de sa communauté ${ }^{25}$, la Cour soulignant, à ce titre, la dualité du droit à la représentation ${ }^{26}$. Elle affirme de la même façon dans sa récente affaire Pacheco León et autres $c$. Honduras que les droits politiques, en général, revêtent "une dimension sociale ${ }^{27}$.

Elle insiste par ailleurs dans son avis $n^{\circ} 22$ sur le fait que si les titulaires des droits et libertés conventionnellement protégés sont des «personnes physiques» et pas morales, «il est nécessaire de préciser que chaque droit implique une analyse spécifique quant à son contenu et sa forme de réalisation ${ }^{28}$. Alors que certains d'entre eux ont directement trait «aux fonctions vitales des êtres humains, ou aux fonctions physiques ou psychologiques du corps humain, comme le droit à la vie, à la liberté ou à l'intégrité personnelle», d'autres, à l'inverse, sont relatifs "à la relation des êtres humains et la société », tels que les droits à la propriété privée, la liberté d'association ou encore le droit à la nationalité 29 .

La Cour en déduit que dans certaines situations les droits et libertés conventionnellement protégés sont exercés au travers d'une personne morale. Aussi, «la reconnaissance de droits [à cette dernière] peut impliquer, de manière directe ou indirecte, la protection des droits de l'homme des personnes physiques associées ${ }^{30}$. Elle affirme alors devoir se livrer à une analyse au cas par cas en vue de savoir «si effectivement la personne physique a exercé son droit au travers de la fiction de la personne morale $»^{31}$, tout en requérant un lien « essentiel et direct» entre les deux ${ }^{32}$. Or, il semble que ce soit bien le cas s'agissant des partis politiques, la Cour affirmant très clairement au détour d'une autre affaire relative cette fois-ci aux moyens de communication que «les partis politiques sont l'instrument permettant aux citoyens d'exercer leurs droits politiques ${ }^{33}$. Ces derniers sont donc considérés comme le moyen, voire la condition d'exercice de ces droits.

Néanmoins, si cela permet à la Cour de reconnaître qu'ils doivent faire l'objet d'une attention particulière et que la dimension sociale de ces droits est également violée, cela ne saurait emporter, pour autant, une reconnaissance de la qualité de victime de ladite personne morale ni une quelconque indemnisation. Dans sa récente affaire Lagos del Campo c. Pérou, relative à la violation des droits d'un représentant d'une organisation syndicale, elle reconnaît par exemple que les deux dimensions de la liberté d'association sont bien atteintes ${ }^{34}$ mais souligne, au stade des réparations, qu'en vertu de l'article 35.1 de son règlement, seul le représentant syndical peut être reconnu comme «victime des violations reconnues dans la présente décision » et, par conséquent, être «bénéficiaire des réparations ordonnées par la Cour ${ }^{35}$.

C'est sans doute pourquoi on décompte si peu d'affaires relatives aux partis politiques en tant que tels

21. Voir M. Rota, L'interprétation des Conventions..., p. 90-97.

22. Cour IDH, Huilca Tecse c. Pérou, fond, réparations et coûts, 3 mars 2005, série C, n $121, \$ 70-72$ et Cour IDH, Cantoral Huamaní et García Santa Cruz c. Pérou, exceptions préliminaires, fond, réparations et coûts, 10 juillet 2007, série C, $\mathrm{n}^{\circ} 167, \$ 148, \$ 162$. Voir aussi l'avis Cour IDH, Titularidad de derechos de las personas jurídicas..., $\$ 110$.

23. Cour IDH, Lagos del Campo c. Pérou, exceptions préliminaires, fond, réparations et coûts, 31 août 2017, série C, $\mathrm{n}^{\circ} 340, \$ 162$. Voir aussi Cour IDH, Chitay Nech et autres $c$. Guatemala, exceptions préliminaires, fond, réparations et coûts, 25 mai 2010, série C, $n^{\circ} 212, \$ 115$.

24. Cour IDH, Lagos del Campo c. Pérou, \$162.

25. Cour IDH, Chitay Nech et autres c. Guatemala, $\$ 113$

26. Ibid., $\$ 115$.

27. Cour IDH, Pacheco León et autres c. Honduras, fond, réparations et coûts, 15 novembre 2017, série C, $\mathrm{n}^{\circ} 342, \S 148$.

28. Cour IDH, Titularidad de derechos de las personas jurídicas..., $\$ 110$.

29. Ibid.

30. Ibid., $\$ 111$.

31. Ibid., $\$ 118$.

32. Ibid., $\$ 119$.

33. Cour IDH, Granier et autres (Radio Caracas Televisión) c. Venezuela, exceptions préliminaires, fond, réparations et coûts, 22 juin 2015 , série C, $\mathrm{n}^{\circ} 293, \$ 148$.

34. Cour IDH, Lagos del Campo c. Pérou, $\$ 162$

35. Ibid., $\$ 196$. 
devant la Cour interaméricaine. Car, il faut le rappeler, la saisine de la Commission nécessite bien souvent, pour qu'elle ait une chance d'aboutir, le soutien d'une organisation non gouvernementale (ONG), apportant un soutien à la fois matériel et juridique aux victimes. Le contentieux soumis à la Cour interaméricaine par la Commission ${ }^{36}$ est, notamment de ce fait, toujours nettement marqué par des violations "graves et systématiques ${ }^{37}$ des droits et libertés. Dès lors et malgré une nette diversification ces dernières années ${ }^{38}$, très peu d'affaires traitent directement cette question.

La Cour a tout de même eu l'occasion de souligner l'importance du rôle joué par les partis politiques au travers d'affaires de disparitions forcées par exemple en affirmant que «les voix de l'opposition sont indispensables dans une société démocratique», puisque nécessaires au débat ${ }^{39}$, et que «la participation effective des individus, groupes et organisations et partis politiques d'opposition dans une société démocratique doit être garantie par les États ${ }^{40}$. Elle ne s'est en revanche directement saisie de la question de leur rôle qu'au travers de trois décisions: les deux premières sont relatives à l'affiliation obligatoire à un parti politique comme condition d'éligibilité ${ }^{41}$ et la troisième concerne plus généralement la restriction des droits "de nature politique ${ }^{42}$ des magistrats et magistrates ${ }^{43}$.

Or, la protection offerte aux partis politiques par la Cour dans ces trois décisions est très en retrait au regard de ce que laissait présager la rédaction des quelques articles de la Convention y ayant indirectement trait - qu'il s'agisse de la liberté d'association ou encore des droits politiques. Elle accepte, tout d'abord, de reconnaître implicitement la licéité des candidatures indépendantes (I). Elle refuse, ensuite, de reconnaître une quelconque exclusivité des partis dans la présentation de candidats à des élections (II). Elle semble enfin annoncer, dans une affaire plus récente, l'importation du concept de démocratie militante (III).

\section{La reconnaissance implicite de la licéité des candidatures indépendantes}

L'affaire Castañeda Gutman c. Mexique interpelle par sa spécificité dans le contentieux interaméricain à plusieurs titres. Il s'agit tout d'abord d'une affaire « que l'on pourrait qualifier de "classique [...]", au sens où [elle serait] l'apanage de n'importe quelle "société démocratique" ${ }^{44}$. La nature des droits en cause - les droits politiques - diffère en effet de ceux invoqués dans la plupart des autres affaires soumises à la Cour. La nature des violations examinées s'en distingue également en ce sens qu'elles ne sauraient être qualifiées de "graves", «systématiques», " massives» ou encore «structurelles» ${ }^{45}$. La victime - candidat indépendant à l'élection présidentielle du Mexique de 2006 - n'appartient pas en outre, selon les critères établis par la Cour, à un quelconque groupe vulnérable. C'est d'ailleurs l'une des rares affaires dans lesquelles la victime se saisit directement du droit de recours individuel sans que le soutien d'une ONG soit rendu nécessaire.

Dans cette affaire, la Cour ne traite qu'indirectement du rôle joué par les partis politiques. Elle commence par souligner l'importance des droits politiques, en général, qui «favorisent le renforcement de la démocratie et du pluralisme politique $»^{46}$. Elle considère cependant que leur application peut être soumise à certaines conditions ${ }^{47}$. Les droits politiques ne sont pas absolus et l'État a la faculté de les réguler ou de les restreindre, dans le respect néanmoins des limites posées par la Convention ${ }^{48}$. Pour être considérées comme compatibles avec la Convention, les restrictions aux droits et libertés doivent respecter certains critères issus de sa jurisprudence classique: ceux de légalité, de finalité légitime, de nécessité et de proportionnalité. Or, c'est uniquement au stade de l'examen de ce dernier qu'apparaît la question du rôle joué par les partis politiques.

36. Dans le système interaméricain de protection des droits et libertés, la Commission interaméricaine joue en effet encore le rôle de filtre. L'article 61.1 de la Convention américaine dispose que «les États parties à la présente Convention et la Commission ont qualité pour saisir la Cour ». Dès lors, dans le cadre d'un recours individuel, seul l'État concerné par la pétition ainsi que la Commission ont la possibilité de saisir le juge, tout comme ce fut le cas sous l'empire de la Convention européenne avant l'entrée en vigueur du Protocole ${ }^{\circ} 11$.

37. M. Rota, L'interprétation des Conventions..., p. 99-101 et 109-112.

38. Ibid., p. 112-116.

39. Cour IDH, Manuel Cepeda Vargas c. Colombie, $\$ 173$. La Cour se réfère également à la jurisprudence de la Cour européenne, notamment aux affaires suivantes: Cour EDH, 8 décembre 1999, ÖZDEP c. Turquie, n² 23885/94, \$ 41; Cour EDH, 25 mai 1998, Parti socialiste et autres c. Turquie, $\mathrm{n}^{\circ} 20 / 1997$ et $804 / 1007, \$ 47$.

40. Cour IDH, Yatama c. Nicaragua, $\$ 201$; avis sur Condición jurídica y derechos de los migrantes indocumentados, $\$ 89$.

41. Cour IDH, Yatama c. Nicaragua et Cour IDH, Castañeda Gutman c. Mexique, exceptions préliminaires, fond et réparations, 6 août 20o8, série $\mathrm{C}, \mathrm{n}^{\circ} 184$.

42. Cette expression est employée par Yannick Lécuyer pour désigner les droits qui «exercent une influence décisive sur la forme du régime politique» (Y. Lécuyer, L'européanisation..., p. 19) et qui englobent donc, notamment, en plus des droits politiques stricto sensu, la liberté d'expression, d'association et de réunion par exemple.

43. Cour IDH, López Lone et autres c. Honduras, exceptions préliminaires, fond, réparations et coûts, 5 octobre 2015 , série C, $\mathrm{n}^{\circ} 302$.

44. L. Burgorgue-Larsen, «Les nouvelles tendances dans la jurisprudence de la Cour interaméricaine des droits de l'homme», in Cursos de derecho internacional y relaciones y internacionales de Vitoria-Gasteiz 2008, Bilbao, Universidad del Pais Vasco, 2009, p. 149-180, spéc. p. 154.

45. Sur ces qualifications, voir M. Rota, L'interprétation des Conventions..., p. 99-101 et 106-112.

46. Cour IDH, Castañeda Gutman c. Mexique, \$141.

47. Sur ce point, voir Cour IDH, Yatama c. Nicaragua, $\$ 206$.

48. Elle cite à cet égard l'article 29.a in fine selon lequel aucune norme qui est issue de la Convention ne peut être interprétée de façon à limiter les droits davantage qu'elle ne le prévoit elle-même (Cour IDH, Castañeda Gutman c. Mexique, $\$ 174$ ). 
Pour caractériser l'exigence de proportionnalité, la Cour cherche en effet à vérifier «si la restriction satisfait une nécessité sociale impérieuse, c'est-à-dire si elle poursuit un intérêt public impératif, restreint le moins possible le droit en cause et est strictement ajustée à l'atteinte de l'objectif légitime ${ }^{49}$. À ce stade, elle examine le système électoral en cause et reconnaît l'existence de deux types de systèmes relatifs aux candidatures aux fonctions politiques justifiés par les besoins démocratiques: ceux qui prévoient la possibilité de se présenter de manière indépendante et ceux qui prévoient l'affiliation obligatoire à un parti ${ }^{50}$. Or, en l'espèce, ce second choix découle de «nécessités sociales impérieuses fondées sur différentes raisons historiques, politiques et sociales $»^{51}$ qui répondent donc à "un intérêt public impératif " ${ }^{52}$. La Cour souligne en effet que le droit international - et donc la Convention - n'impose pas de système électoral déterminé ${ }^{53}$ ni de modalités précises de mise en œuvre des droits de vote et d'éligibilité54 ${ }^{54}$ Elle se réfère en outre au "droit électoral comparé » des États de «la région» pour démontrer l'existence de deux systèmes juridiques potentiellement compatibles avec la Convention ${ }^{55}$. Il existe, selon elle, un certain équilibre entre les deux systèmes évoqués dans la mesure où, lorsque la possibilité d'inscrire des candidats indépendants est prévue en droit interne, des exigences équivalentes à celles que requiert l'affiliation obligatoire à un parti politique sont instaurées en parallèle. Elle relève par exemple l'obligation faite au candidat de justifier d'un nombre ou pourcentage d'électeurs appuyant sa candidature, ce qui implique que la procédure électorale soit organisée de manière efficace (système prévu au Chili, en Équateur, au Honduras, au Pérou, au Venezuela). La présentation de programmes politiques pour toute la durée du mandat auquel il se présente, des garanties économiques ou encore une orga- nisation particulière proche de celles de partis peuvent en outre parfois être exigées ${ }^{56}$. Elle en conclut alors qu'aucun des deux systèmes ne restreint, en soi, le droit d'être élu, consacré dans l'article 23 de la Convention. Refusant d'effectuer un contrôle "in abstracto" en la matière ${ }^{57}$, elle laisse alors le soin à l'État d'organiser son propre système électoral ${ }^{58}$.

Elle insiste à ce titre sur l'existence d' « une profonde crise reliée aux partis politiques, au pouvoir législatif et aux personnes qui dirigent les sujets publics » et sur la

[...] nécessité impérieuse d'un débat profond et réfléchi sur la participation et la représentation politique, la transparence et le rapprochement des institutions des personnes, en définitive, sur le renforcement et l'approfondissement de la démocratie ${ }^{59}$.

Or, il revient à l'État et à la société civile de développer une telle réflexion et d'effectuer des propositions pour y parvenir. Les États doivent aussi privilégier les mesures qui permettent de renforcer les droits politiques et la démocratie, et ce au regard de leur propre «développement historique et politique ${ }^{60}$. Les candidatures indépendantes peuvent être considérées comme un moyen d'atteindre ce but, «entre beaucoup d'autres " ${ }^{61}$, la Cour reconnaissant implicitement que l'État est le mieux placé pour consacrer le système pertinent au regard de l'intérêt général.

La Cour n'impose donc pas un régime uniforme et fait simplement en sorte que le standard conventionnel minimum soit respecté ${ }^{62}$. Sa démarche s'approche donc de l'harmonisation des droits internes, chère à la Cour européenne et qui dénote avec celle que la Cour interaméricaine adopte habituellement. Il faut, pour le comprendre, rappeler que la Cour interaméricaine rejette par principe le concept de «marge nationale d'appréciation »,

49. Cour IDH, Castañeda Gutman c. Mexique, $\$ 186$.

50. Il s'agit en effet de permettre un accès direct aux fonctions politiques à tout citoyen, de renforcer les partis politiques reconnus comme étant des instruments fondamentaux pour la démocratie ou d'organiser de manière efficace la procédure électorale (Cour IDH, Castañeda Gutman c. Mexique, $\$ 192$ ).

51. Cour IDH, Castañeda Gutman c. Mexique, $\$ 193$, à savoir: «La nécessité de créer et renforcer le système des partis envisagé en tant que réponse à une réalité historique et politique; la nécessité d'organiser de manière efficace la procédure électorale dans une société de plus de 75 millions d'électeurs, au sein de laquelle tous ont le droit d'être élus; la nécessité d'un système de financement essentiellement public pour assurer le développement d'élections libres et authentiques, dans des conditions égales; et la nécessité de contrôler de manière effective les fonds utilisés pour les élections» (ibid.).

52. Ibid.

53. Ibid., $\$ 166$.

54. Ibid., $\$ 162$. La Cour reconnaît l’existence de différents systèmes compatibles avec la Convention (ibid., $\$ 173$ ), solution qui est saluée par la doctrine. Voir, par exemple, C. M. Pelayo Möller, S. J. Vázquez Camacho, «El caso Castañeda ante la Corte Interamericana de Derechos Humanos», Anuario Mexicano de Derecho Internacional, vol. 9, 2009, p. 808.

55. Cour IDH, Castañeda Gutman c. Mexique, $\$ 198$.

56. Ibid., $\$ 199$.

57. Ibid., $\$ 200$.

58. Elle précise néanmoins que c'est à la condition que le droit d'être élu soit accessible à tous dans des conditions d'égalité (ibid., $\$ 201$ ).

59. Cour IDH, Castañeda Gutman c. Mexique, \$204.

60. Ibid.

61. Ibid.

62. Ibid., $\$$ 166. Parmi ces règles minimales se trouve l'obligation faite aux États de mettre en place un système électoral prévoyant des élections périodiques, authentiques, par le biais du suffrage universel et équitable, à bulletin secret et qui garantit la libre expression de la volonté des électeurs (ibid., $\$ 158)$. Les États ont donc la possibilité de réglementer la participation politique mais seulement lorsque ces règles sont « raisonnables au regard des principes de la démocratie représentative» (ibid., §149). 
largement développé par la Cour européenne ${ }^{63}$. Son interprétation restrictive du caractère subsidiaire de la Convention l'amène aussi à généralement adopter des solutions beaucoup plus tranchées ${ }^{64}$. L'affaire Castañeda Gutman c. Mexique fait donc encore une fois figure d'exception, jouant ici en la défaveur des partis politiques. Car laisser le soin aux États de décider des moyens en vue de renforcer les droits politiques et la démocratie aboutit à ce que la Cour reconnaisse la licéité potentielle des candidatures indépendantes.

Aucune exclusivité ne saurait donc être reconnue aux partis politiques s'agissant de la présentation de candidats à d'éventuelles élections. Ils peuvent être concurrencés par l'action d'un individu seul. Mais ils peuvent l'être aussi par d'autres formes d'organisations ayant aussi cette vocation et dont la légitimité est reconnue par la Cour.

\section{La reconnaissance d'autres formes d'organisation politique aussi légitimes que les partis}

Dans l'affaire Yatama c. Nicaragua, la Cour a été confrontée à une situation a priori similaire puisque la loi nicaraguayenne imposait pareillement une condition d'affiliation à un parti politique pour pouvoir présenter une candidature aux élections municipales. En l'espèce, les victimes, membres d'une communauté autochtone, se sont bien conformées à cette exigence ${ }^{65}$ en créant spécialement le parti YATAMA, acronyme de Yapti Tasba Masraka Nanih Asla Takanka (Organisation des enfants de la terre mère), regroupant les représentants de plusieurs communautés désignés selon les principes de la "démocratie communautaire ${ }^{66}$. Ce parti n'a cependant pas pu présenter de candidats à des élections municipales en raison d'une loi électorale restrictive. Cette dernière imposait aux partis politiques d'inscrire des candidats dans au moins $80 \%$ des communes et sur au moins $80 \%$ des postes à pourvoir $^{67}$. Faute de candidats suffisants sur l'ensemble de la circonscription concernée, YATAMA s'est vu refuser leur inscription sur les listes électorales.
S'agissant de la question de l'affiliation obligatoire à un parti politique, la Cour l'examine à l'aune de son test de compatibilité des restrictions avec la Convention, tout en insistant sur le caractère non discriminatoire qu'elle doit revêtir ${ }^{68}$. Elle souligne en effet que les droits politiques doivent être garantis par l'État « dans des conditions d'égalité ${ }^{69}$ et lit l'article 23 de manière combinée avec les articles 1.1 et 24 relatifs au principe d'égalité et à la non-discrimination. Or, on le rappelle, la Cour entend le principe d'égalité non pas comme l'égalité devant la loi mais comme l'égalité réelle, dite encore substantielle ${ }^{70}$. Pour l'atteindre, elle impose alors aux États d'adopter des mesures spécifiques en vue de prendre en considération «la situation de fragilité ou de détresse dans laquelle se trouvent les membres de certains secteurs ou groupes sociaux $»^{71}$. Aussi, les minorités, dans la mesure où elles sont dans une position de vulnérabilité caractérisée, doivent pouvoir bénéficier de mesures spécifiques, sans quoi leurs droits politiques sont violés ${ }^{72}$, ce qui était le cas en l'espèce.

La Cour commence en effet par affirmer qu'aucune disposition de la Convention ne saurait impliquer l'affiliation obligatoire à un parti politique pour se porter candidat à une quelconque élection ${ }^{73}$. Malgré l'importance que les partis politiques revêtent, elle reconnaît qu'il existe «d'autres formes d'organisations au travers desquelles des candidatures à des charges électives ont pour but la réalisation de fins communes », et leur reconnaît une influence particulière «lorsque cela est pertinent et même nécessaire pour favoriser et assurer la participation politique de groupes spécifiques de la société ${ }^{74}$. Cette participation d'autres organisations que les partis est même considérée comme « essentielle» en vue de permettre à un groupe de citoyens d'exercer son droit à la participation 75 . Sans elle, il deviendrait donc ineffectif.

En l'espèce, même si les populations en cause se sont bien pliées à cette exigence de constitution d'un parti politique, celle-ci les a contraintes à former une «organisation éloignée de leurs usages, coutumes et traditions » ${ }^{76}$ et a atteint le droit à la participation desdits candidats aux élections municipales. Or, l'État n'a pas démontré que cette restriction poursuivait « un but utile et opportun, qui

63. Voir, par exemple, Cour EDH, 2 mars 1987, Mathieu Mohin et Clerfayt c. Belgique, $\mathrm{n}^{\circ}$ 9267/81, $\$ 52$ et 54 . Voir également l'affaire Podkolzina c. Lettonie, dans laquelle la Cour invoque pareillement les «facteurs historiques et politiques propres à chaque État » ainsi que «la diversité des choix possibles en la matière» (Cour EDH, 9 avril 2002, Podkolzina c. Lettonie, 46726/99, $\$ 33$ ).

64. Voir M. Rota, L'interprétation des Conventions..., p. 212-215.

65. Cour IDH, Yatama c. Nicaragua, $\$ 124.28$ et 214.

66. Cette expression renvoie, selon les requérants, à une forme d'organisation «héritée de leurs ancêtres» et «fondée sur des assemblées de communautés et de quartiers sur les territoires indigènes ou ethniques ou régionaux» (ibid., \$124.13).

67. Ibid., $\$ 221$

68. Ibid., $\$ 206$.

69. Ibid., $\$ 194$. Aussi, les citoyens doivent pouvoir élire librement et de manière égale leurs représentants (ibid., $\$ 198)$, mais également candidater à des charges électorales dans des conditions d'égalité (ibid., \$206).

70. M. Rota, L'interprétation des Conventions..., p. 393.

71. Cour IDH, Yatama c. Nicaragua, $\$ 101$.

72. Ibid., $\$ 201$.

73. Ibid., \$215.

74. Ibid.

75. Ibid., $\$ 217$.

76. Ibid., $\$ 218$. 
la rendrait nécessaire pour satisfaire à un intérêt public impératif ${ }^{77}$. Elle a, à l'inverse, aboutit à ce que les membres de la communauté autochtone qui ont intégré YATAMA soient empêchés d'exercer pleinement leur droit d'être élu $^{78}$. La Cour considère alors cette restriction à l'exercice d'un droit politique comme incompatible avec la Convention ${ }^{79}$. Se livrant toujours à une lecture combinée des droits politiques avec le principe d'égalité, elle ajoute que les exigences liées à la participation politique, conçues pour les partis politiques, et qui ne peuvent pas être respectées par des regroupements organisés différemment, sont aussi contraires à la Convention ${ }^{80}$.

Cette lecture combinée des droits politiques avec le principe d'égalité a cependant des conséquences paradoxales. Car une même restriction aux droits politiques - le fait d'imposer une condition d'affiliation à un parti peut en effet être, ou non, considérée comme une violation de l'article 23 en fonction de son titulaire. Dans l'affaire Castañeda Gutman c. Mexique, la Cour constate que la victime ne représente pas les intérêts d'un quelconque groupe vulnérable ou marginalisé qui serait de facto privé de l'accès au droit d'éligibilité et qu'elle a eu la possibilité de s'affilier à un parti ${ }^{81}$. Dans la mesure où cette restriction remplit bien les critères de légalité, de finalité légitime, de nécessité et de proportionnalité, elle considère qu'elle est légitime et que les droits politiques n'ont donc pas été violés $^{82}$. Dans l'affaire Yatama $c$. Nicaragua, à l'inverse, elle insiste sur le fait que les victimes sont des membres de communautés autochtones «qui se différencient de la majorité de la population » et qui «affrontent de sérieuses difficultés qui les maintiennent dans une situation de vulnérabilité et de marginalité ${ }^{83}$. Aussi, l'État doit prendre en considération «leurs valeurs, usages, coutumes, rites et formes d'organisation ${ }^{84}$ et reconnaître d'autres formations que les partis politiques ${ }^{85}$. La jurisprudence de la Cour interaméricaine se distingue de ce point de vue clairement de celle de la Cour européenne, qui s'inscrit dans une vision beaucoup plus libérale des droits et liber- tés, construite sur « une conception générale et absolue du statut de l'individu qui rejette le différentialisme au nom de l'homogénéité du corps social ${ }^{86}$.

Elle s'en éloigne aussi s'agissant de l'examen de la seconde exigence, celle faite aux partis de présenter des candidats dans au moins $80 \%$ des communes de la circonscription visée et sur au moins $80 \%$ des postes à pourvoir. Cela a contraint, selon la Cour, YATAMA à présenter des candidats dans des communes au sein desquelles aucun habitant n'était membre de ces communautés autochtones, d'où une difficulté réelle d'en trouver ${ }^{87}$. La Cour en conclut qu'il s'agit d'une restriction elle aussi inconventionnelle puisque disproportionnée, ayant limité indûment la participation politique des candidats proposés par YATAMA aux élections municipales ${ }^{88}$. L'État devait en effet prendre en considération le fait que la population autochtone était minoritaire dans la circonscription en cause et l'absence d'appui potentiel qu'elle pouvait trouver dans certaines communes ${ }^{89}$.

Le raisonnement de la Cour interaméricaine est donc une fois encore bien éloigné de celui de la Cour européenne développé dans l'affaire Partei Die Friesen c. Allemagne par exemple. En l'espèce, cette dernière reconnaît en effet la situation de «désavantage» dans laquelle se trouvent les partis représentant « des groupes d'intérêt numériquement faibles définis par des critères tels que l'âge, la conviction religieuse ou la profession ${ }^{9 \circ}$ - pour le dire autrement, des minorités. Elle considère en revanche que cette situation résulte $\mathrm{du}$ "choix» du parti en cause de ne représenter "que les intérêts d'une petite partie de la population ${ }^{91}$ et que l'État ne peut de ce fait pas en être «tenu pour responsable $»^{92}$. Dès lors, et même si la Cour reconnaît par ailleurs que la constitution d'une association, et donc d'un parti, peut permettre à une minorité de préserver et de défendre ses droits ${ }^{93}$, elle reprend le principe dégagé par la Commission selon lequel «la Convention n'oblige pas les Parties contractantes à avantager les minorités » ${ }^{94}$. De plus, malgré l'adoption de la Convention-cadre pour

81. Cour IDH, Castañeda Gutman c. Mexique, $\$ 172$.

82. Ibid.

83. Cour IDH, Yatama c. Nicaragua, $\$ 202$.

84. Ibid., $\$ 225$.

85. Ibid., $\$ 215-217$.

86. Y. Attal-Galy, Droits de l'homme..., p. 2.

87. Cour IDH, Yatama c. Nicaragua, \$ 221-223.

88. Ibid., $\$ 223$.

89. Ibid.

90. Cour EDH, $5^{\mathrm{e}}$ section, 28 janvier 2016, Partei Die Friesen c. Allemagne, $\mathrm{n}^{\mathrm{o}} 6548 \mathrm{o} / 10, \$ 40$ (nous traduisons).

91. Ibid.

92. Ibid.

93. La Cour rappelle le principe dégagé dans l'affaire Gorzelik et autres c. Pologne selon lequel «la fondation d'une association afin d'exprimer et promouvoir l'identité d'une minorité peut aider cette dernière à préserver et défendre ses droits » (Cour EDH, GC, 17 février 2004, Gorzelik et autres c. Pologne, $\mathrm{n}^{\circ}$ 44158/98, \$ 93; voir aussi Cour EDH, $5^{\mathrm{e}}$ section, 28 janvier 2016, Partei Die Friesen c. Allemagne, $\left.\$ 41\right)$.

94. Com. EDH, déc., 15 avril 1996, Magnago et Südtiroler Volkspartei c. Italie, nº 25035/94; cité par Cour EDH, $5^{\mathrm{e}}$ section, 28 janvier 2016, Partei Die Friesen c. Allemagne, $\$ 42$. Notons que, dans la version anglaise de ces affaires, la Cour affirme, à la suite de la Commission, que la Convention n'impose pas aux États de prévoir des «discriminations positives» en faveur des minorités: "the Convention does not compel the Contracting Parties to provide for positive discrimination in favour of minorities» (ibid.). 
la protection des minorités nationales ${ }^{95}$ et le fait qu'elle encourage la mise en place de mécanismes permettant une participation effective des minorités aux affaires publiques, les juges de Strasbourg se rallient à la position de la Cour constitutionnelle de Basse-Saxe selon laquelle il ne découle de cette Convention « aucune obligation claire et contraignante» d'exempter les partis représentant des minorités nationales de l'obligation d'atteindre un certain seuil de voix pour obtenir un mandat ${ }^{96}$. Aussi, même interprétée à la lumière de ce texte, «la Convention ne requiert pas un traitement différent en faveur de partis minoritaires ${ }^{97}$.

On le voit, la philosophie sociale des droits et libertés dans laquelle s'inscrit la jurisprudence de la Cour de San José heurte de plein fouet la philosophie libérale défendue par la Cour de Strasbourg ${ }^{98}$. Cette différence de conception aboutit en outre à des solutions clairement opposées. Cela pourrait aussi être le cas s'agissant de l'importation du concept de démocratie militante dans la jurisprudence de la Cour interaméricaine.

\section{L'importation annoncée du concept de démocratie militante}

La Cour interaméricaine n'a jamais été amenée à se prononcer directement sur des restrictions aux droits adoptées en vue de défendre la démocratie entendue dans son sens libéral, qui place les droits et libertés au fondement du pouvoir ${ }^{99}$. La Cour européenne, quant à elle, n'a pas hésité à importer le concept de démocratie militante, développé notamment par le constitutionnaliste allemand Karl Loewenstein ${ }^{100}$. Ce dernier, «prétendant dépasser la tension considérée jusqu'alors insoluble entre liberté des citoyens et sûreté de l'État ${ }^{101}$, prône la mise en place de «mécanismes de défense active pour éviter que la démocratie et les principes constitutionnels ne soient mis en péril par l'exercice des droits et libertés ${ }^{102}$. Ces idées ont clairement influencé les rédacteurs de la Convention européenne ${ }^{103}$ qui consacre, dans son article 17, l'interdiction de l'abus de droit ${ }^{104}$.

Sans pour autant se fonder sur cette disposition, la Cour a consacré le principe dit de « démocratie apte à se défendre » invoqué par certains États européens ayant connu des régimes totalitaires pour justifier certaines restrictions aux droits et libertés conventionnellement protégés. La Cour s'en remet par exemple à «l'expérience que [l'Allemagne] a connue sous la République de Weimar» et au «cauchemar du nazisme » ${ }^{105}$, ou encore au «contexte historique précis» que connaît la Hongrie ${ }^{106}$, au "passé politique» de la Lettonie ${ }^{107}$ et au «régime théocratique islamique» qui a sévi en Turquie ${ }^{108}$, pour permettre aux États d'adopter des restrictions aux droits de nature politique des requérants en vue de sauvegarder certains principes démocratiques. Les restrictions sont considérées comme nécessaires à la protection du caractère démocratique de la société, dans la mesure où il a déjà été atteint par le passé.

La célèbre affaire Refah Partisi c. Turquie est intéressante du point de vue du sujet traité puisque la restriction en cause concernait justement la dissolution d'un parti politique prônant la mise en place d'un régime politique fondé sur la Charia. Soulignant, entre autres, l'incompatibilité de ces principes avec ceux de la démocratie ${ }^{109}$, la Cour refuse de considérer cette restriction à la liberté d'association comme contraire à la Convention. Elle souligne à ce titre

[...] qu'un parti politique dont les responsables incitent à recourir à la violence ou proposent un projet politique qui ne respecte pas la démocratie ou qui vise la destruction de celle-ci ainsi que la méconnaissance des droits et libertés qu'elle reconnaît, ne peut se prévaloir de la protection de la Convention contre les sanctions infligées pour ces motifs ${ }^{110}$.

Elle consacre donc la vision la plus radicale de la démocratie militante puisqu'aboutissant à un encadrement non

95. La Convention-cadre pour la protection des minorités nationales, élaborée par la Commission de Venise et adoptée le $1^{\text {er }}$ février 1995 dans le cadre du Conseil de l'Europe, est entrée en vigueur le $1^{\text {er }}$ février 1998 après douze ratifications.

96. Cour EDH, $5^{\mathrm{e}}$ section, 28 janvier 2016, Partei Die Friesen c. Allemagne, $₫ 43$.

97. Ibid.

98. M. Rota, L'interprétation des Conventions..., p. 368-371.

99. En ce sens, la démocratie est entendue comme régime politique au sein duquel le peuple est le détenteur du pouvoir. Il ne doit donc obéir qu'aux normes qu'il a lui-même adoptées d'où la nécessaire autonomie qui lui est reconnue et dont il va jouir via la reconnaissance par l'État de droits et libertés. Sur ce point, voir, entre autres, M.-A. Cohendet, Droit constitutionnel, $3^{\mathrm{e}}$ éd., Issy-les-Moulineaux, LGDJ (Cours), 2017, p. 31-32.

100. Sur les origines du concept, voir A. Simard, "L'échec de la Constitution de Weimar et les origines de la "démocratie militante" en RFA", Jus Politicum, $\mathrm{n}^{\circ}$ 1, décembre 2008, en ligne: http://juspoliticum.com/article/L-echec-de-la-Constitution-de-Weimar-et-les-origines-de-lademocratie-militante-en-R-F-A-29.html.

101. Ibid.

102. Ü. Kilinç, «La conception de la démocratie militante dans la jurisprudence de la Cour européenne des droits de l'homme», Revue trimestrielle des droits de l'homme, nº 90, 2012, p. 298.

103. Ibid., p. 299-301.

104. Selon cet article, en effet, "Aucune des dispositions de la présente Convention ne peut être interprétée comme impliquant pour un État, un groupement ou un individu, un droit quelconque de se livrer à une activité ou d'accomplir un acte visant à la destruction des droits ou libertés reconnus dans la présente Convention ou à des limitations plus amples de ces droits et libertés que celles prévues à ladite Convention ».

105. Cour EDH, GC, 26 septembre 1995, Vogt c. Allemagne, nº 17851/91, \$ 51.

106. Cour EDH, GC, 20 mai 1999, Rekvényi c. Hongrie, $\mathrm{n}^{\circ} 25390 / 94, \$ 48$.

107. Cour EDH, GC, 16 mars 2006, Ždanoka c. Lettonie, $\mathrm{n}^{\circ}$ 58278/00, $\$ 83$.

108. Cour EDH, GC, 13 février 2003, Refah Partisi (Parti de la prospérité) et autres c. Turquie, no 41340/98, 41342/98, 41343/98 et 41344/98, \$ 125.

109. Ibid., $\$ 123$.

110. Ibid., $\$ 98$. 
seulement des activités des partis politiques mais aussi des idées ${ }^{111}$.

Le continent américain a lui aussi été influencé par la pensée des émigrés allemands à partir de 1933, et notamment par celle de Karl Loewenstein ${ }^{112}$. On trouve d'ailleurs dans le Pacte de San José une clause d'abus de droit beaucoup plus détaillée que celle figurant au sein de la Convention de Rome $^{113}$. La Cour interaméricaine, quant à elle, ne s'est pas encore directement saisie de ce concept. Sa décision Yatama c. Nicaragua laisse cependant présager sa reprise dans un sens aussi radical que celui qui est adopté par la Cour européenne puisqu'elle précise que les partis politiques et les organisations ou groupes qui participent à la vie de l'État «doivent avoir des buts compatibles avec le respect des droits et libertés conventionnellement protégés ${ }^{114}$.

Elle le développe en outre dans sa récente décision López Lone et autres c. Honduras mais l'aborde néanmoins différemment. Il était question, dans cette affaire, du devoir de réserve des juges honduriens, restreignant leur droit de "participer à la direction des affaires publiques ${ }^{115}$. La Cour reconnaît tout d'abord que les droits politiques revêtent un caractère fondamental dans une "société démocratique», tout comme d'ailleurs la liberté d'expression et de réunion. Ces droits ne sont cependant pas absolus et peuvent faire l'objet de restrictions, dans la mesure où celles-ci respectent les différents critères de compatibilité avec la Convention ${ }^{116}$.

Mais la Cour souligne aussi que des restrictions différentes de celles classiquement admises peuvent être envisageables s'agissant des magistrats et magistrates en raison de leurs fonctions en tant que tels. Elles ne le sont cependant que "dans les conditions normales de l'État de droit ${ }^{117}$ et se justifient par le souci de garantir l'indépendance et l'impartialité des juges, par ailleurs consacrées par différents textes et organes internationaux sur lesquels la Cour s'appuie explicitement ${ }^{118}$. De plus, et dans la mesure où l'article 8 de la Convention américaine consacre le droit d'être entendu devant un juge «indépendant et impartial», elle considère qu'il s'agit bien d'un objectif légitime permettant de restreindre leurs droits ${ }^{119}$. Il existe, enfin, un «consensus régional relatif à la nécessité de restreindre les activités politiques et partisanes des juges, sachant que dans certains États, toute participation en politique est, d'une manière générale, prohibée, excepté le vote aux élections ${ }^{120}$.

Néanmoins, la Cour rappelle aussitôt que «la faculté pour l'État de réguler ou de restreindre ces droits n'est pas discrétionnaire et que toute limitation aux droits consacrés par la Convention doit s'interpréter de manière restrictive ${ }^{121}$. Aussi, les restrictions «à la participation d'activités de type partisane des juges» ne sauraient être « interprétée[s] de façon à ce qu'elles ne les empêchent de participer à toute discussion de nature politique ${ }^{122}$.

C'est ce qui justifie que les juges doivent pouvoir se réapproprier leurs droits politiques et donc sortir de leur devoir de réserve en cas de situation de mise en péril de la démocratie. La Cour précise en effet qu'il y a des cas dans lesquels un juge, en tant que citoyen, "considère qu'il a un devoir moral de s'exprimer ${ }^{123}$. Elle en déduit que les normes qui restreignent la participation politique des juges ne sont pas applicables dans le cadre d'agissements en défense de l'ordre démocratique ${ }^{124}$. Le fait de ne pas leur laisser la possibilité de se prononcer contre un coup d'État serait même contraire à «l'indépendance entre les pouvoirs de l'État » et à ses «obligations internationales » telles qu'elles découlent de sa qualité de membre de l'Organisation des États américains (OEA) ${ }^{125}$.

De telles circonstances invalident donc toute procédure disciplinaire menée à leur encontre, pourtant jugées compatibles avec la Convention dans des circonstances normales. En période de "graves crises démocratiques", l'exercice de leur liberté d'expression, de leur droit de réunion et de manifestation doit s'analyser comme un "exercice légitime de leurs droits, en tant que citoyens»"

111. Sur l'évolution de la conception de la démocratie militante avant et après la Seconde Guerre mondiale en ce sens, voir A. Simard, «L'échec de la Constitution de Weimar...».

112. Sur ce point, voir A. Berthout, La démocratie militante, thèse de doctorat en droit public sous la direction de J. Arlettaz, université de Montpellier, en cours de rédaction.

113. L'article 29 de la Convention américaine prévoit en effet qu' "Aucune disposition de la présente Convention ne peut être interprétée comme: a. Autorisant un État partie, un groupement ou un individu à supprimer la jouissance et l'exercice des droits et libertés reconnus dans la présente Convention ou à les restreindre plus qu'il n'est prévu dans ladite Convention; b. restreignant la jouissance et l'exercice de tout droit ou de toute liberté reconnus par la législation d'un État partie ou dans une convention à laquelle cet État est partie; c. excluant d'autres droits et garanties inhérents à la personne humaine ou qui dérivent de la forme démocratique représentative de gouvernement; d. supprimant ou limitant les effets que peuvent avoir la Déclaration américaine des Droits et Devoirs de l'Homme et tous autres actes internationaux de même nature».

114. Cour IDH, Yatama c. Nicaragua, \$216.

115. Article 23.1.a de la Convention américaine.

116. Cour IDH, López Lone et autres c. Honduras, $\$ 168$.

117. Ibid., $\$ 169$.

118. Ibid. $\$ 170$.

119. Ibid., $\$ 171$.

120. Ibid., $\$ 172$.

121. Ibid.

122. Ibid.

123. Ibid., $\$ 173$. On pourrait cependant objecter à la Cour qu'elle se fonde sur tout un ensemble de textes internationaux qui consacrent davantage l'obligation faite aux juges de dénoncer toute atteinte à leur indépendance.

124. Ibid., $\$ 174$.

125. Ibid.

126. Ibid. (nous soulignons). 
Le seul fait d'initier des procédures disciplinaires à leur encontre «suite à leurs actions d'opposition au coup d'État et en faveur de l'État de droit» est d'ailleurs, lui aussi, considéré comme étant contraire à la Convention, au regard de son caractère «intimidant $»^{127}$.

Peut-on cependant en conclure que le juge aurait la possibilité de s'affilier à un parti durant cette période de contestation de l'ordre établi? La question reste posée. On peut en revanche regretter l'assimilation ou plutôt la confusion que semble ici faire la Cour entre théorie de l'État de droit et démocratie. Car si elle insiste bien dans un premier temps sur la nécessité pour les juges de pouvoir s'exprimer en tant que citoyen en période de crise politique pour défendre la démocratie, il ne semble pas que ce soit en faveur de l'expression du pouvoir constituant originaire. Leur action a-t-elle uniquement pour but de protéger l'ordre constitutionnel établi (et donc l'État de droit stricto sensu)? Ou est-ce véritablement la sauvegarde de la démocratie (ce qui supposerait que les juges puissent aussi agir en vue de changer de Constitution) qui est visée? La substitution du terme «État de droit» à celui de «démocratie » à la fin de son raisonnement laisse planer le doute.

Afin de le lever, les juges de San José pourraient très bien reprendre l'argumentaire développé par ceux de Strasbourg dans l'affaire Refah Partisi c. Turquie. Car si ces derniers reconnaissent «qu'un parti politique peut promouvoir un changement de la législation ou des structures légales ou constitutionnelles de l'État », ils affirment qu'il ne peut le faire qu'à deux conditions: «1. les moyens utilisés à cet effet doivent être légaux et démocratiques; 2. le changement proposé doit lui-même être compatible avec les principes démocratiques fondamentaux $»^{128}$. La Cour prône donc un encadrement du pouvoir constituant en vue de permettre le respect des principes fondamentaux de la démocratie libérale qui trouve son fondement dans le respect des droits et libertés.
La reprise de ce concept serait-il cependant de nature à heurter les principes inhérents au concept de «démocratie sociale » que la Cour interaméricaine défend quant à elle? Il semble qu'il faille répondre par la négative. Car il ne se distingue de celui de démocratie libérale qu'au regard des fondements philosophiques des droits et libertés protégés. Alors que les tenants de la démocratie libérale les fondent davantage sur le nécessaire respect de la liberté, ceux de la démocratie sociale insistent quant à eux sur le nécessaire respect de l'égalité ${ }^{129}$. Le fondement de la démocratie reste cependant le même: elle suppose que chacun soit indépendant du pouvoir mais aussi que toute personne ait la possibilité de se l'en approprier, de manière libre et égale ${ }^{130}$. La prise en compte différenciée de la liberté et de l'égalité réelle ou substantielle explique néanmoins que la notion de démocratie puisse être entendue différemment et que les principes qui la sous-tendent ne soient pas les mêmes. C'est alors en cela que la jurisprudence des deux Cours pourrait se distinguer: les principes fondamentaux à défendre pourraient ne pas être si semblables.

Le rôle joué par les partis politiques dans le standard de société démocratique tel que développé par la Cour interaméricaine et au regard duquel elle lit la Convention est bien relatif, notamment lorsqu'on le compare avec celui qui est reconnu au sein de la jurisprudence européenne. Ceci s'explique principalement par son attachement au principe d'égalité, qui peut aussi se manifester à d'autres égards. C'est sur ce fondement que la Cour se saisit, par exemple, des mécanismes de démocratie participative ${ }^{131}$. Elle incite aussi les États parties à la Convention à s'affranchir du modèle classique du régime représentatif classique, au sein duquel les partis politiques jouent un rôle primordial. La jurisprudence de la Cour interaméricaine invite donc une fois encore à la réflexion, particulièrement nécessaire dans nos démocraties libérales souffrant d'une crise du régime représentatif couplée aujourd'hui avec celle des partis.

127. Cour IDH, López Lone et autres c. Honduras, $\$ 176$.

128. Cour EDH, GC, Refah Partisi (Parti de la prospérité) et autres c. Turquie, $\$ 98$.

129. Pour une analyse historique de ce concept en Amérique latine, se référer aux travaux de S. García Ramírez, «Estado democrático y social de derecho", Boletín Mexicano de Derecho Comparado, n 98, 200o, p. 595-635; H. Gros Espiell, Estudios sobre Derechos Humanos, Madrid, Civitas, 1988, t. II, notamment p. 65-86. Voir également les travaux récents des Argentins Víctor Abramovich et Christian Courtis, mais aussi de l'Italien Luigi Ferrajoli auteurs de référence en la matière, notamment V. Abramovich, C. Courtis, El umbral de la ciudadanía. El significado de los derechos sociales en el Estado social constitucional, Buenos Aires, Editores del Puerto, 2006; L. Ferrajoli, "Estado social y Estado de derecho", in Derechos sociales. Instrucciones de uso, V. Abramovich, M. J. Añón, C. Courtis (dir.), Mexico, Fontamara, 20o6, p. 11-21. Pour une analyse de ce concept et son évolution en langue française, se référer à l'intervention de C. M. Herrera, «État social et droits sociaux fondamentaux» au colloque «État et régulation sociale», CES-Matisse, Paris, 11-13 septembre 2006. Voir aussi M. Rota, L'interprétation des Conventions..., p. 348-354 et 365-368.

130. Selon Hans Kelsen, c'est «la synthèse de ces deux principes [liberté et égalité] qui est la caractéristique de la démocratie» (H. Kelsen, La démocratie, sa nature, sa valeur, Paris, Dalloz, 1932, p. 2).

131. Voir, par exemple, Cour IDH, Pueblo Indígena Kichwa de Sarayaku c. Équateur, fond et réparations, 27 juin 2012 , série C, $\mathrm{n}^{\circ} 245, \S 159$. 